\title{
3D Cardiac Deformation from Ultrasound Images
}

\author{
Xenophon Papademetris ${ }^{1}$, Albert J. Sinusas ${ }^{23}$, Donald P. Dione ${ }^{3}$, and \\ James S. Duncan ${ }^{12}$ \\ 1 Departments of Electrical Engineering \\ 2 Diagnostic Radiology \\ 3 Medicine \\ Yale University New Haven, CT 06520-8042 \\ papad@noodle.med.yale.edu
}

\begin{abstract}
The quantitative estimation of regional cardiac deformation from 3D image sequences has important clinical implications for the assessment of viability in the heart wall. Such estimates have so far been obtained almost exclusively from Magnetic Resonance (MR) images, specifically MR tagging. In this paper we describe a methodology for estimating cardiac deformations from 3D ultrasound images. The images are segmented interactively and then initial correspondence is established using a shape-tracking approach. A dense motion field is then estimated using an anisotropic linear elastic model, which accounts for the fiber directions in the left-ventricle. The dense motion field is in turn used to calculate the deformation of the heart wall in terms of strain in cardiac specific directions. The strains obtained using this approach in open-chest dogs before and after coronary occlusion related to changes in blood flow, show good agreement with previously published results in the literature. This proposed method provides quantitative regional 3D estimates of heart deformation from ultrasound images.
\end{abstract}

\section{Introduction}

The fundamental goal of many efforts in the cardiac imaging and image analysis community is to assess the regional function of the left ventricle (LV) of the heart. The general consensus is that the analysis of heart wall deformation provides quantitative estimates of the location and extent of ischemic myocardial injury. There have been considerable efforts within the medical image analysis community aimed at estimating this deformation, almost exclusively using magnetic resonance (MR) images, primarily MR tagging [13,7,11], and to a lesser extent, MR phase contrast $[9,14]$ velocity images.

In this paper we describe and test an approach to estimate the regional deformation of the left-ventricle from ultrasound left ventricular images. We use a biomechanical model to describe the myocardium and shape-based tracking displacement estimates on the epi and endo-cardial walls to generate the initial displacement estimates. These are integrated in a Bayesian estimation 
framework and the overall problem is solved using the finite element method. This method produces quantitative regional 3D cardiac deformation estimates from ultrasound images which up-to now was thought to be only possible using magnetic resonance and especially MR tagging. The fast improving quality of ultrasound images with the introduction of harmonic imaging [6] and contrast agents [15] should make it possible to obtain even more accurate estimates of $3 \mathrm{D}$ left ventricular deformation in the future.

\section{Our Approach}

We estimate a dense displacement field within a Bayesian estimation framework which consists of a data term and a model term. These are described in sections 2.1 and 2.2 respectively.

\subsection{Obtaining Initial Displacement Data}

Image Acquisition The images were acquired using an HP Sonos 5500 Ultrasound System with a 3D transducer (Transthoracic OmniPlane 21349A (R5012)). The 3d-probe was placed at the apex of the left-ventricle of an open-chest dog using a small ultrasound gelpad (Aquaflex) as a standoff as shown in figure 1. Each acquisition consisted of 13-17 frames per cardiac cycle depending on the heart rate. The angular slice spacing was 5 degrees resulting in 36 image slices for each frame.

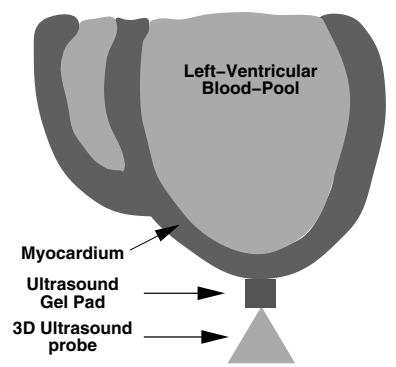

Fig. 1. Image acquisition geometry.

Image Segmentation The endo- and epi- cardial surfaces were extracted interactively using a software platform [16] originally developed for MR image data and subsequently modified to allow for the different geometry and image characteristics of ultrasound. For the automated part of the segmentation, for each image slice, we used an integrated deformable boundary method whose external energy function consisted of the standard intensity term and a texturebased term similar to the integrated method proposed in [3], although in our case the contours were parameterized as b-splines to allow for easy interaction. 


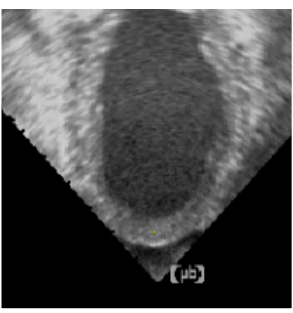

Original Image

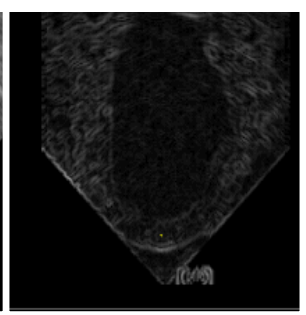

Texture Based MRF Segmentation
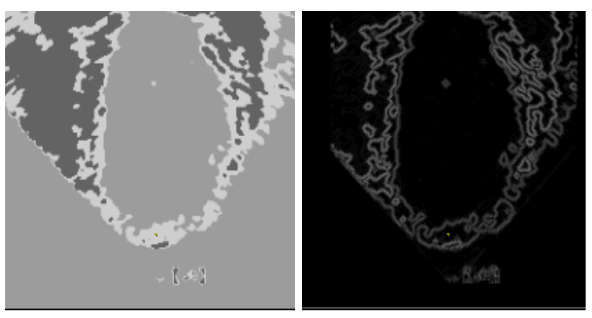

Texture+Intensity Intensity Energy Map Energy Map

Fig. 2. External Energy Functions for intensity and intensity+texture snakes. Note that the intensity only energy function is very noisy inside the leftventricular blood-pool which creates many local minima for the deformable contour. The use of the texture eliminates most of these minima.

This produced reasonable results as shown in figures 2 and 3. We are currently looking into more sophisticated techniques for generating the external energy maps, including those suggested by Mulet-Parada et al [10].

Shape-Tracking Displacement Estimates In this work, the original displacements on the outer surfaces of the myocardium were obtained by using the shape-tracking algorithm whose details were presented in [12]. The method tries to track points on successive surfaces using a shape similarity metric which tries to minimize the difference in principal curvatures and was validated using implanted markers [12].

For example, consider point $p_{1}$ on a surface at time $t_{1}$ which is to be mapped to a point $p_{2}$ on the deformed surface at time $t_{2}$. First, a search is performed a physically plausible region $W_{2}$ on the deformed surface and the point $\hat{p}_{2}$ which has the local shape properties closest to those $p_{1}$ is selected. The shape properties here are captured in terms of the principal curvatures $\kappa_{1}$ and $\kappa_{2}$. The distance measure used is the bending energy required to bend a curved plate or surface patch to a newly deformed state. This is labeled as $d_{b e}$ and is defined as:

$$
d_{b e}(p 1, p 2)=A\left(\frac{\left(\kappa_{1}\left(p_{1}\right)-\kappa_{1}\left(p_{2}\right)\right)^{2}+\left(\kappa_{2}\left(p_{1}\right)-\kappa_{2}\left(p_{2}\right)\right)^{2}}{2}\right)
$$

The displacement estimate vector for each point $p_{1}, u_{1}^{m}$ is given by

$$
u_{1}^{m}=\hat{p}_{2}-p_{1} \quad, \quad \hat{p}_{2}=\underset{p_{2} \in W_{2}}{\arg \min }\left[d_{b e}(p 1, p 2)\right]
$$

Confidence Measures in the match: The bending energy measures for all the points inside the search region $W_{2}$ are recorded as the basis to measure the goodness and uniqueness of the matching choices. The value of the minimum 


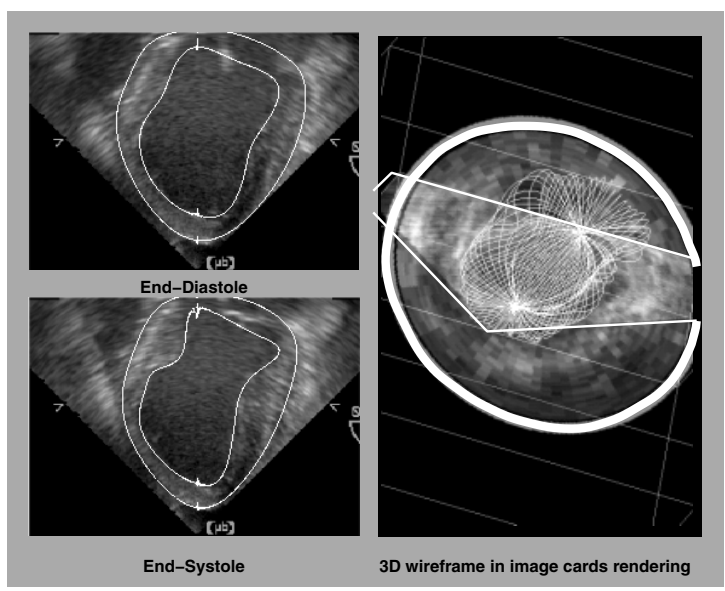

Fig. 3. Images and superimposed extracted contours. Only two of the eight frames are shown. The 3D rendering on the right shows all the wire-frame contours superimposed on a long axis (original) and a short-axis (interpolated) image slices.

bending energy in the search region between the matched points indicates the goodness of the match. Denote this value as $m_{g}$, we have the following measure for matching goodness:

$$
m_{g}\left(p_{1}\right)=d_{b e}\left(p_{1}, \hat{p}_{2}\right)
$$

On the other hand, it is desirable that the chosen matching point is a unique choice among the candidate points within the search window. Ideally, the bending energy value of the chosen point should be an outlier (much smaller value) compared to the values of the rest of the points. If we denote the mean values of the bending energy measures of all the points inside window $W_{2}$ except the chosen point as $\bar{d}_{b e}$ and the standard deviation as $\sigma_{b e}^{d}$, we define the uniqueness measure as:

$$
m_{u}\left(p_{1}\right)=\frac{d_{b e}\left(p_{1}, \hat{p}_{2}\right)}{\bar{d}_{b e}-\sigma_{b e}^{d}}
$$

This uniqueness measure has a high value if the bending energy of the chosen point is small compared to some smaller value (mean minus standard deviation) of the remaining bending energy measures. Combining these two measures together, we arrive at one confidence measure $c^{m}\left(p_{1}\right)$ for the matched point $\hat{p}_{2}$ of point $p_{1}$ :

$$
c^{m}\left(p_{1}\right)=\frac{1}{k_{1, g}+k_{2, g} m_{g}\left(p_{1}\right)} \times \frac{1}{k_{1, u}+k_{2, u} m_{u}\left(p_{1}\right)}
$$

where $k_{1, g}, k_{2, g}, k_{1, u}$, and $k_{2, u}$ are scaling constants for normalization purposes. We normalize the confidences to lie in the range 0 to 1 . 
Modeling the initial displacement estimates: Given a set of displacement vector measurements $u^{m}$ and confidence measures $c^{m}$ we model theses estimates probabilistically by assuming that the noise in the individual measurements us normally distributed with zero mean and a variance equal to $\frac{1}{c^{m}}$. In addition we assume that the measurements are uncorrelated, although this is an assumption that is not strictly correct and we are presently working on this. Given these assumptions we can write the measurement probability for each point as:

$$
p\left(u^{m} \mid u\right)=\frac{1}{\sqrt{2 \pi \sigma^{2}}} e^{\frac{\left(u-u^{m}\right)^{2}}{2 \sigma^{2}}}
$$

\subsection{Modeling the Myocardium}

The passive properties of the left-ventricular myocardium are captured using a biomechanical model. We use an anisotropic linear elastic model which allows us to incorporate information about the preferential stiffness of the tissue along fiber directions from [5]. These fiber directions are shown in figure 4. The model described in terms of an internal or strain energy function of the form:

$$
W=\epsilon^{\prime} C \epsilon
$$

where $\epsilon$ is the strain and $C$ is the $6 \times 6$ matrix containing the elastic constants which define the material properties. This is described in more detailed in continuum mechanics textbooks such as Malvern [8].

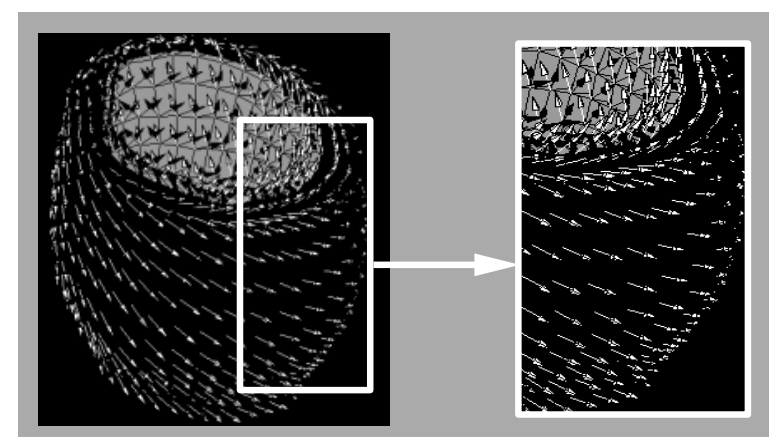

Fig. 4. Fiber direction in the left ventricle as defined in Guccione et al [5].

Deformation and Strain: Consider a body $B(0)$ which after time $t$ moves and deforms to body $B(t)$. A point $\mathrm{X}$ on $B(0)$ goes to a point $x$ on $B(t)$ and the transformation gradient $F$ is defined as $d x=F d X$. The deformation is expressed in terms of the strain tensor $\epsilon$. Because the deformations to be estimated in this work are larger than 5\%, we use a finite strain formulation implemented using a logarithmic strain $\epsilon^{L}$, which is defined as: $\epsilon=\ln \sqrt{F \cdot F^{\prime}}$. Since the strain tensor is a $3 \times 3$ symmetric 2 nd-rank tensor (matrix), we can re-write it in vector form as, $e=\left[\begin{array}{llllll}\epsilon_{11} & \epsilon_{22} & \epsilon_{33} & \epsilon_{12} & \epsilon_{13} & \epsilon_{23}\end{array}\right]^{\prime}$. This will enable us to express the tensor equations in a more familiar matrix notation. 
Strain Energy Function: The mechanical model can be defined in terms of a strain energy function. The simplest useful continuum model in solid mechanics is the linear elastic one which is of the form: $W=e^{\prime} C e$ where $C$ is a $6 \times 6$ matrix and defines the material properties of the deforming body. The left ventricle of the heart is specifically modeled as a transversely elastic material to account for the preferential stiffness in the fiber direction, using the matrix $C$ :

$$
C^{-1}=\left[\begin{array}{cccccc}
\frac{1}{E_{p}} & \frac{-\nu_{p}}{E_{p}} & \frac{-\nu_{f p}}{E_{f}} & 0 & 0 & 0 \\
\frac{-\nu_{p}}{E_{p}} & \frac{1}{E_{p}} & \frac{-\nu_{f p}}{E_{f}} & 0 & 0 & 0 \\
\frac{-\nu_{f p} E_{f}}{E_{p}} & \frac{-\nu_{f p} E_{f}}{E_{p}} & \frac{1}{E_{f}} & 0 & 0 & 0 \\
0 & 0 & 0 & \frac{2\left(1+\nu_{p}\right)}{E_{p}} & 0 & 0 \\
0 & 0 & 0 & 0 & \frac{1}{G_{f}} & 0 \\
0 & 0 & 0 & 0 & 0 & \frac{1}{G_{f}}
\end{array}\right]
$$

where $E_{f}$ is the fiber stiffness, $E_{p}$ is cross-fiber stiffness and $\nu_{f p}, \nu_{p}$ are the corresponding Poisson's ratios and $G_{f}$ is the shear modulus across fibers. $\left(G_{f} \approx\right.$ $E_{f} /\left(2\left(1+\nu_{f p}\right)\right)$. If $E_{f}=E_{p}$ and $\nu_{p}=\nu_{f p}$ this model reduces to the more common isotropic linear elastic model. The fiber stiffness was set to be 3.5 times greater than the cross-fiber stiffness [5]. The Poisson's ratios were both set to 0.4 to model approximate incompressibility. This model was previously used in [17].

A probabilistic description of the model: As previously demonstrated by Christiansen et al [4] there is a correspondence between an internal energy function and a Gibbs-Prior. If the mechanical model is described in terms of an internal energy function $W(C, u)$, where $C$ represents the material properties and $u$ the displacement field, then we can write an equivalent prior probability density function $p(u)$ (see equation 9) of the Gibbs form:

$$
p(u)=k_{1} \exp (-W(C, u))
$$

\subsection{Integrating the Data and Model Terms}

Having defined both the data term model (equation 5) and the model term (equation 8) in terms of probability density functions we naturally proceed to write the overall problem in a Bayesian estimation framework as follows: Given a set of noisy input displacement vectors $u^{m}$ and the associated noise model $p\left(u^{m} \mid u\right)$ (data term) and a prior probability density function $p(u)$ (model term), find the best output displacements $\hat{u}$ which maximize the posterior probability $p\left(u \mid u^{m}\right)$. Using Bayes' rule we can write.

$$
\hat{u}=\underset{u}{\arg \max } p\left(u \mid u^{m}\right)=\underset{u}{\arg \max }\left(\frac{p\left(u^{m} \mid u\right) p(u)}{p\left(u^{m}\right)}\right)
$$

The prior probability of the measurements $p\left(u^{m}\right)$ is a constant once these measurements have been made and therefore drops out of the minimization process. 
Taking logarithms in equation (9) and differentiating with respect to the displacement field $u$ results in a system of partial differential equations, which we solve using the Finite Element Method [2]. The first step in the finite element method is the division or tessellation of the body of interest into elements; these are commonly tetrahedral or hexahedral in shape. Once this is done, the partial differential equations are written down in integral form for each element, and then the integral of these equations over all the elements is taken to produce the final set of equations. For more information one is referred to standard textbooks such as Bathe [2]. The final set of equations is then solved to produce the output set of displacements. In our case the myocardium is divided into approximately 2,500 hexahedral elements.

For each frame between end-systole (ES) and end-diastole (ED), a two step problem is posed: (i) solving equation (9) normally and (ii) adjusting the position of all points on the endo-and epi-cardial surfaces so they lie on the endo- and epicardial surfaces at the next frame using a modified nearest-neighbor technique and solving equation (9) once more using this added constraint. This ensures that there is no bias in the estimation of the radial strain.

\section{Results}

To evaluate the efficacy of using image-derived in vivo deformation estimates to measure regional LV function we conducted experiments on fasting, anesthetized, open chest, adult mongrel dogs with approval of the Yale University Animal Care and Use Committee. In this preliminary work, we report results from three animals. The 3D ultrasound images were obtained either before (dog1) or after occlusion of the left anterior descending coronary artery ( $\operatorname{dog} 2$ and dog3). Coronary occlusion created an area of dysfunction which we call the risk area. Also regional blood flow in the heart wall was determined using a radio-labeled microsphere technique. Here, radioactively labeled microspheres were injected into the left atrium and reference blood samples were drawn from the femoral arteries. Regional myocardial blood flow was calculated using a method previously described in Sinusas et al [1]. The blood flow measurements are used to identify the risk area and play no further role in this work.

The images were segmented interactively and the surfaces sampled to 0.5 voxel resolution, at which point curvatures were calculated and the shapetracking algorithm was used to generate initial displacement estimates. The heart wall was divided into 2500 hexahedral elements and the anisotropic linear elastic model was used to regularize the displacements. The computational time after the segmentation was of the order of 3-4 hrs/dog (depending on the heart rate and hence the number of image frames) on a Silicon Graphics Octane with an R10000 195 Mhz processor and 128 MB RAM,

For the purpose of analyzing the results, the left-ventricle of the heart was divided into 4 cross-sectional slices, slice 1 being at the bottom or apex of the ventricle and number 4 being at the top or base of the ventricle. Each slice was further subdivided into 8 sectors, as shown in figure 5. A sector was la- 
beled as being in the risk area if the endocardial mircrosphere flow was less than $0.25 \mathrm{ml} / \mathrm{min} / \mathrm{g}$. The normal region was defined by 5 transmural sectors located in the posterior lateral wall at the base of the heart (sectors 5,6,7 of the basal slice and sectors 6,7 of the mid-basal slice). We report the average of radial and circumferential strains for the risk areas and the normal regions.

\begin{tabular}{|l|c|c|c|c|c|c|}
\hline Study & Norm RR \% & Risk RR \% & Norm CC \% & Risk CC \% & Norm LL \% & Risk LL \% \\
\hline $\operatorname{dog} 1$ & 17.7 & n/a & -13.4 & n/a & -4.3 & n/a \\
\hline $\operatorname{dog} 2$ & 22.4 & -4.3 & -8.4 & 1.9 & -3.4 & -0.7 \\
\hline $\operatorname{dog} 3$ & 17.2 & -13.7 & -12.4 & -7.3 & -3.1 & -2.0 \\
\hline
\end{tabular}

Table 1. Summary of results for three animal studies (Norm=Normal Region, Risk $=$ Risk Area, $\mathrm{RR}=$ Average Radial Strain, $\mathrm{CC}=$ Average Circumferential Strain, LL=Average Longitudinal Strain).

The results are summarized in Table 1. Function in the risk area, which was independently defined by microsphere flow, was markedly reduced compared to non-affected regions and the control normal animal. The radial strain is notably smaller in the risk area after coronary occlusion. The circumferential strain becomes less negative also indicating a loss of function. There was a small decrease in the longitudinal strain as well. The progressive development of regional radial and circumferential strains for 'dog2' is shown in figure 6 .

Croisille et al [11] reported similar values (Radial $=23.2 \%$, Circum. $=-10.5 \%$ and Long. $=-7.5 \%$ ) for strains in the normal regions of dog hearts using threedimensional tagged MRI. However, they observed smaller reductions in strains post-occlusion, which can be attributed to coronary re-perfusion in their model and significantly delayed imaging after the occlusion (2 days later as opposed to 15-20 minutes in our case). This probably allowed for partial recovery of function in the risk region.

\section{Conclusions}

In this work we have demonstrated that estimates of $3 \mathrm{D}$ cardiac deformation can be obtained from ultrasound images, which are consistent with regional blood flow measurements. The estimated strains are also consistent with values reported in the literature using MR tagging [11]. In the future we hope to validate these estimates of regional deformation directly by comparing them to strains concurrently measured from implanted sonomicrometers.

\section{Acknowledgments}

The first author would like to thank Farah Janzad for her help with segmenting the images. Additional thanks to Jason Soares and Jennifer $\mathrm{Hu}$ for tissue processing and surgical preparation respectively. We also would like to acknowledge support from the National Institutes of Health and the American Heart Association. 


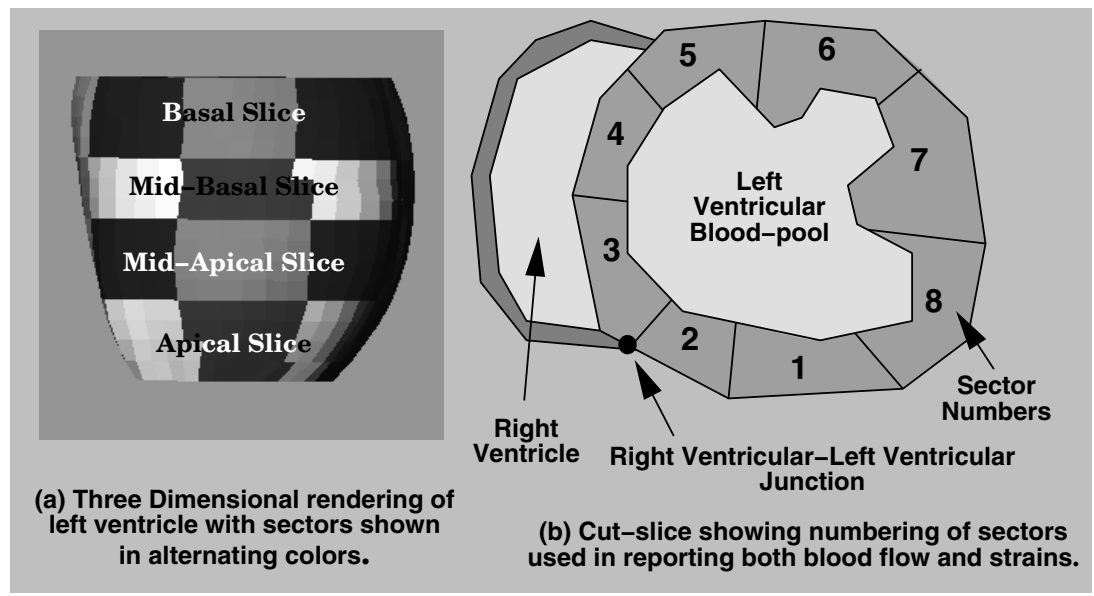

Fig. 5. Division of a slice of the heart for the purpose of reporting results. Each sector consists of approximately 75 elements in the finite element mesh.

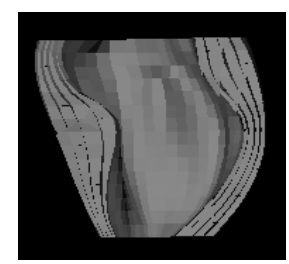

End-Diastole

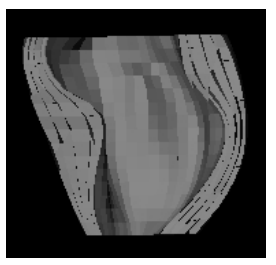

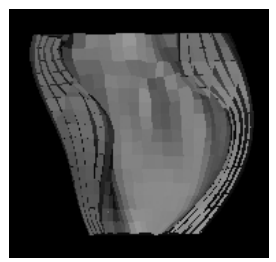
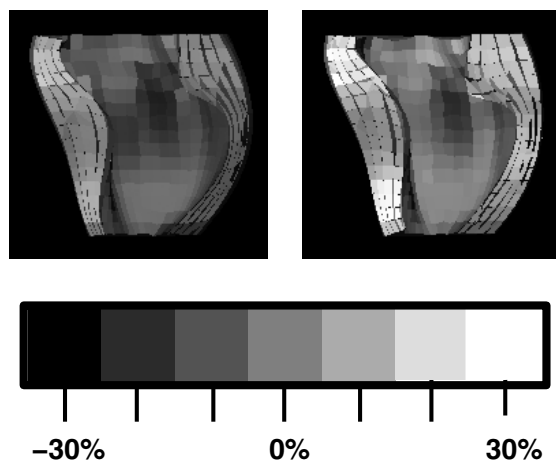

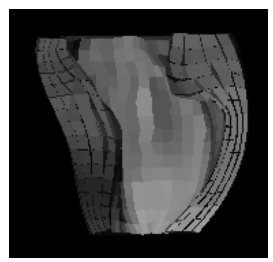

End-Systole
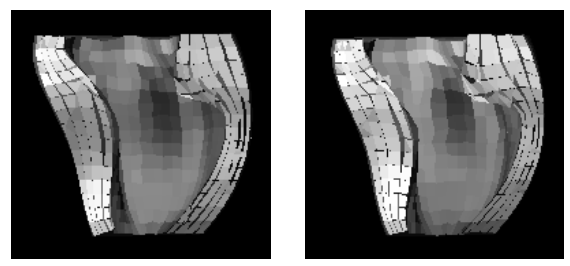

Fig. 6. A long-axis cut-away sectional view of the left ventricle showing circumferential(top) and radial(bottom) strain development in a dog following left anterior descending coronary artery occlusion (on the lower right half of the heart). Note the normal behavior in the left half of the heart. There was positive radial strain (thickening) and negative circumferential strain (shortening) as we move from End Diastole to End Systole. The lower right half of the heart where the affected region was located showed almost the opposite behavior, as expected. 


\section{References}

1. Sinusas A.J., Trautman K.A., Bergin J.D., Watson D.D., Ruiz M., Smith W.H., and Beller G.A. Quantification of area of risk during coronary occlusion and degree of myocardial salvage after reperfusion with technetium-99m methoxyisobutyl isonitrile. Circulation, 82:1424-37, 1990. 426

2. K. Bathe. Finite Element Procedures in Engineering Analysis. Prentice-Hall, New Jersey, 1982. 426

3. A. Chakraborty, M. Worring, and J.S. Duncan. On multi-feature integration for deformable boundary finding. Proceedings of the International Conference on Computer Vision, pages 846-851, 1995. 421

4. Christiansen G. E., Rabbitt R. D., and Miller M. I. 3D brain mapping using deformable neuroanatomy. Physics in Medicine and Biology, 39:609-618, 1994. 425

5. J. M. Guccione and A. D. McCulloch. Finite element modeling of ventricular mechanics. In P. J. Hunter, A. McCulloch, and P. Nielsen, editors, Theory of Heart, pages 122-144. Springer-Verlag, Berlin, 1991. 424, 425

6. Caidahl K., Kazzam E., Lingberg J., Andersen G. N., Nordanstig J., Dahlqvist S.R., Waldenstro A, and Wikh R. New concept in echocardiography: harmonic imaging of tissue withoug use of contrast agent. The Lancet, 352:1264-1270, 1999. 421

7. Prince J. L. and McVeigh E. R. Motion estimation from tagged mr image sequences. IEEE Transactions on Medical Imaging, 11:238-249, June 1992. 420

8. Lawrence E. Malvern. Introduction to the Mechanics of a Continuous Medium. Prentice-Hall, Englewood Cliffs, New Jersey, 1969. 424

9. F. G. Meyer, R. T. Constable, A. G. Sinusas, and J. S. Duncan. Tracking myocardial deformation using spatially constrained velocities. In Information Processing in Medical Imaging. Kluwer, 1995. 420

10. Mulet-Parada Miguel and Noble J. Alison. 2d+t acoustic boundary detection in echocardiography. In MICCAI, Boston, Massachusetts, October 1998. 422

11. Croissile P., Moore C.C., Judd R.M., Lima J.A.C, Arai M., McVeigh E.R., Becker L.C., and Zerhouni E.A. Differentiation of viable and nonviable myocardium by the use of three-dimensional tagged mri in 2-day-old reperfused infarcts. Circulation, 99:284-291, 1999. 420, 427

12. Shi P., Sinusas A.J., Constable R.T., Ritman E., and Duncan J.S. Point-tracked quantitative analysis of left ventricular motion from $3 \mathrm{D}$ image sequences. IEEE Transactions on Medical Imaging, in-press. 422

13. J. Park, D. Metaxas, and L. Axel. Volumetric deformable models with parameter functions: a new approach to the 3D motion analysis of the LV from MRI-SPAMM. In Fifth International Conference on Computer Vision, pages 700-705, 1995. 420

14. P. Shi, G. Robinson, A. Chakraborty, L. Staib, R. T. Constable, A. Sinusas, and J. Duncan. A unified framework to assess myocardial function from $4 \mathrm{D}$ images. In Lecture Notes in Computer Science: First International Conference on Computer Vision, Virtual Reality, and Robotics in Medicine, pages 327-337, 1995. 420

15. Porter T.R., Xie F., , Kricsfeld A., Chiou A., and Dabestani A. Improved endocardial border resolution using dobutamin stress endocardiography with intravenous sonicated dextrose albumin. J. Am College of Cardiology, 23:1440-43, 1994. 421 
16. Papademetris X., Rambo J., Dione D.P., Sinusas A.J., and Duncan J.S. Visually interactive cine-3D segmentation of cardiac mr images. Suppl. to the J. Am. Coll. of Cardiology Vol. 31, Number 2 (Suppl. A), February 1998. 421

17. Papademetris X., Shi P., Dione D.P., Sinusas A.J., and Duncan J.S. Recovery of soft tissue object deformation using biomechanical models. In Information Processing in Medical Imaging, Visegrad, Hungary, June 1999. 425 